\title{
Relações entre cultura e tecnologia: uma abordagem sobre o sistema da Moda
}

\author{
Relation between culture and technology: \\ an approach about the Fashion system
}

GIOTTI, Josiane; Mestranda em Design; Centro Universitário Ritter dos Reis

josiane.giotti@gmail.com

PARODE, Fábio Pezzi; Doutor em Estética; Centro Universitário Ritter dos Reis

fparode@gmail.com

RIBEIRO, Vinicius Gadis; Doutor em Computação; Centro Universitário Ritter dos Reis

vinicius@uniritter.edu.br

\section{Resumo}

O presente artigo investiga quais são as relações entre o sistema da Moda, a cultura e a tecnologia. A relevância da pesquisa se dá ao observar que ainda não existem análises com esta temática específica no país. A metodologia empregada na pesquisa é a qualitativa, sendo utilizado o método descritivo para a sua realização. Para a análise foram abordadas reflexões teóricas sobre o funcionamento da indústria cultural, a sociedade de consumo e qual é a influência da cultura na tecnologia. Posteriormente foi analisado como funciona o sistema da Moda como fenômeno social, cultural, de consumo e a relação positiva e negativa da tecnologia em seus processos de pesquisa, produção e comercialização. Após a análise descritiva, identificou-se que é possível a interação e harmonia entre estes complexos e paradoxos campos da cultura e da tecnologia diante do sistema da Moda.

Palavras Chave: cultura; tecnologia; sistema da Moda.

\section{Abstract}

This article investigates the relationships between the Fashion system, culture and technology. The relevance of the research is given by noting that there are still no analyzes with this specific theme in the country. The methodology used in the research is the qualitative one, being used the descriptive method for its accomplishment. For the analysis, theoretical reflections on the functioning of the cultural industry, the consumer society and the influence of culture on technology were discussed. Later it was analyzed how the fashion system works as a social, cultural, consumer phenomenon and the positive and negative relation of technology in its research, production and commercialization processes. After the descriptive analysis, it was identified that it is possible the interaction and harmony between these complex and paradoxical fields of culture and technology before the Fashion system.

Keywords: culture; technology; Fashion system. 


\section{INTRODUÇÃO}

O presente artigo analisa quais são as relações entre a Moda, a cultura do consumo e a tecnologia. Também, investiga como o sistema da Moda pode se beneficiar da tecnologia, de forma a otimizar os processos produtivos agregando valor aos produtos produzidos, levando em consideração ainda a individualidade dos sujeitos. A relevância da pesquisa se dá ao observar que ainda não existem análises com essa temática específica no Brasil, visto que as reflexões realizadas podem gerar discussões complementares futuras.

Na pesquisa sobre o estado da arte, utilizou-se como aparato os textos de Baudrillard (2008) sobre a sociedade de consumo e de Adorno e Horkheimer (1985) para contextualizar os aspectos da indústria cultural, além de Murphie e Potts (2003) no que se refere à influência da tecnologia na cultura. No estudo sobre o sistema da Moda como fenômeno social e cultural, contou-se com a contribuição de Simmel (2005) e Lipovetsky (2009) e em relação ao sistema da Moda e o consumo utilizou-se as publicações de Conti (2008) e Treptow (2013). Para referenciar a relação entre o sistema da Moda e a tecnologia, utilizou-se como respaldo os textos de Araújo, Neves e Neves (2008) e novamente de Treptow (2013). Para esclarecer a metodologia de pesquisa utilizada, o estudo foi baseado em Sampieri, Collado e Lucio (2013). Para tanto, a metodologia utilizada para realizar a pesquisa é a qualitativa e o método utilizado para a análise foi o descritivo. O foco da pesquisa se dá por meio de reflexões teóricas e análise das relações que ocorrem entre o sistema da Moda, o consumo e a tecnologia (SAMPIERI; COLLADO; LUCIO, 2013).

Para apresentar o estudo, expôs-se na seção 2, expor quais são as relações entre consumo, cultura e tecnologia. Na seção 3 analisa-se como se dá o funcionamento do sistema da Moda, dividido nas subseções 3.1, é apresentada a discussão sobre o sistema da Moda como fenômeno social, cultural e de consumo e na subseção 3.2, discorre-se como o sistema da Moda utiliza a tecnologia em seus processos de pesquisa, produção e comercialização de produtos. Posteriormente, na seção 4, é descrita detalhadamente a metodologia utilizada na pesquisa e realizada a análise da relação entre moda, consumo e tecnologia. E, por fim, as considerações finais são apresentadas na seção 5.

\section{CULTURA, CONSUMO E TECNOLOGIA}

Baudrillard (2008) é um dos principais pensadores pós-modernos que discute a sociedade de consumo, termo este que é utilizado nas áreas relacionadas à sociologia e à economia e surgiu a partir da Revolução Industrial. O conceito trouxe à tona as características de uma sociedade que possui um grande desenvolvimento industrial capitalista e que se caracteriza pelo consumo massivo de bens e serviços. Assim, é por meio deles que a sociedade de consumo se comunica, na medida em que estes se tornaram a sua principal linguagem, a linguagem dos signos.

O aparecimento desse novo sistema de valores, pertencente ao mundo capitalista, é sustentado pela comunicação de massa, feita por meio da publicidade, que geralmente exclui a cultura e o saber dos indivíduos. O resultado deste processo de comunicação, na maioria das vezes, faz com que os sujeitos não consumam por necessidade e bem-estar, mas por exigência de 
aceitação e pertencimento a um grupo ou por status, assim, o consumo surge como comportamento ativo e coletivo, que compõe um sistema de valores de integração de grupos e de controle social.

Neste contexto, o processo de escolha inconsciente e automática do consumidor tem como consequências a aceitação do estilo de vida de determinada sociedade em particular, o que não consiste mais em uma escolha individual e autônoma dos indivíduos, trazendo a discussão do conceito de cultura de massas. Assim, entende-se que a cultura já não é produzida para durar, pois em virtude do modo de produção, encontra-se submetida à mesma função que os bens materiais. Desta forma, os objetos culturais perdem todo o significado humano quando passa a não existir mais distinção entre objetos culturais e materiais (BAUDRILLARD, 2008).

Os sociólogos alemães Adorno e Horkheimer (1985) também discutem o conceito de cultura de massas por meio da crítica à indústria cultural. Esse movimento levou a arte ao nível do consumo e à massificação do gosto, assim, "a técnica da indústria cultural levou apenas à padronização e a produção em série, sacrificando o que fazia diferença entre a lógica da obra e a do sistema social" (ADORNO; HORKHEIMER, 1985, p. 114).

O desenvolvimento da indústria cultural possibilitou um controle e domínio completos sobre os indivíduos, assim, impulsos de transformação e reflexões sobre o mundo não acorrem por meio da indústria cultural, ao contrário disto, seu papel no mundo de superficialidade é evitar todos os impulsos que poderiam formar um sujeito ativo e racional. Este processo de anulação dos indivíduos ficou ainda mais perceptível a partir da chegada da tecnologia como meio de divulgação e consumo de bens e serviços à sociedade. Murphie e Potts (2003) explicam o impacto cultural gerado pela tecnologia perante os indivíduos e os grupos sociais, ressaltando que a cultura e a tecnologia têm uma relação complexa, capaz de gerar discussões que incorporam muitas áreas de interesse em um campo fluido e efêmero.

A tecnologia, impulsionada pela revolução industrial, trouxe como sua principal contribuição à sociedade a ampliação da capacidade produtiva de artefatos por meio das indústrias dos mais diversos segmentos, criando assim, a sociedade industrial em que se vive atualmente. A produção em massa de automóveis criou uma cultura de passageiros e turistas. 0 rádio criou uma cultura de músicos e de ouvintes, assim como a televisão criou a cultura dos cinemas, das novelas, das notícias constantes, dos reality shows e das propagandas. Neste contexto se deu o surgimento da nova arte da mídia, também chamada de multimídia, cibercultura e mídia digital. Basicamente tudo voltado à cultura do consumo (MURPHIE; POTTS, 2003).

$\mathrm{Na}$ contemporaneidade, percebe-se nitidamente que o importante não é a própria tecnologia, mas o sistema social ou econômico no qual ela está inserida. Em um momento em que as novas tecnologias desempenham um papel proeminente da propriedade intelectual, vem à tona à mudança da noção de comunidade, assim, observa-se que os sujeitos se tornaram totalmente submissos às tecnologias e consequentemente também, à cultura do consumo. A tecnologia é utilizada em praticamente todos os setores da indústria e comércio de bens e serviços, inclusive no campo do Design e da Moda. Nas próximas seções, serão apresentadas as discussões sobre o sistema da Moda como fenômeno social, cultural e de consumo, além do panorama da utilização da tecnologia na indústria da Moda. 


\section{O SISTEMA DA MODA}

A Moda não está relacionada somente aos artefatos produzidos por sua indústria, mas é considerado um sistema complexo em que ocorrem fenômenos culturais, midiáticos, consumistas e produtivos, definidos por processos projetuais. Neste contexto, basicamente todos os processos dependem do uso da tecnologia, especialmente os que estão ligados à produção, consumo e distribuição de produtos.

\subsection{O sistema da Moda como fenômeno social, cultural e de consumo}

Para Simmel (2005) e Lipovetsky (2009), a Moda é um fenômeno social que funciona por meio de um sistema efêmero, de fantasia estética e baseado no individualismo. A Moda é um sistema helicoidal de mudanças, pois nada é duradouro, preza-se pelo novo e moderno, onde há uma ruptura com o passado. Os autores entendem a Moda como um fenômeno de estetificação, onde reside um paradoxo entre imitação e distinção, existindo a necessidade do outro para que haja a expressão da individualidade do sujeito. Simmel (2005) por sua vez, não compreende a Moda somente no âmbito individual, mas destaca a ideia de pertencimento do sujeito à um grupo, o que traz à tona a noção da importância das diferentes classes sociais, existindo vigorosamente um movimento de imitação das classes superiores por parte das classes inferiores e da busca constante de diferenciação feita novamente pelas elites sociais.

Conti (2008), baseada nas teorias de Simmel (2005) e Lipovetsky (2009) reafirma a ideia de que a moda retrata o espírito do tempo (zeitgeist), representando cada vez mais a condição social da moda, expressa por meio das mudanças sociais, econômicas, políticas e culturais. Assim, é possível considerar a Moda como um importante fenômeno de cultura e consumo de massa, que representa as mutações e desejos coletivos. Consequentemente, é possível compreender que o complexo sistema da Moda é organizado, baseando-se na história de Moda, em uma lógica social, industrial e tecnológica, dividido em três importantes fases: alta-costura, o prêt-à-porter e o postfashion. Esse sistema estabelece uma relação contínua entre vida social, gostos individuais e coletivos e, mercado produtor (CONTI, 2008).

Em conformidade com Treptow (2013), o sistema tradicional de Moda chamado de altacostura, está relacionado à confecção de peças feitas artesanalmente, com exclusividade e com as medidas de cada cliente. Fischer (2010) sustenta que a origem da alta-costura se deu no século XVIII, tendo o seu nascimento atribuído à costureira Rose Bertin, chapeleira da arquiduquesa Maria Antonieta e ao estilista Charles Frederick Worth, que iniciaram o processo de criar os modelos conforme as suas próprias ideias. Anteriormente ao surgimento da técnica, as peças de vestuário eram feitas por costureiros conforme os pedidos das clientes.

O sistema da alta-costura teve o seu auge entre os anos de 1858 e 1968, mas atualmente ainda ganha lugar de destaque no mundo da Moda, por meio de estilistas franceses, com peças produzidas nos ateliês em Paris que atendem em torno de 200 clientes no mundo todo. Com tal característica, o desfile de alta-costura vislumbra mais a divulgação das marcas e a venda de peças não exclusivas do que a real comercialização das peças únicas (TREPTOW, 2013). 
Em contraponto, o sistema de Moda chamado prêt-à-porter (pronto para vestir) surgiu logo após a Segunda Guerra Mundial. Logo ficou popular por proporcionar mais praticidade e variedade na compra de peças de vestuário, além é claro, de ter preços mais acessíveis do que as peças feitas sob medida por um costureiro ou estilista. A produção de peças feitas em escala industrial reforça a ideia da produção para suprir os desejos coletivos (TREPTOW, 2013). Atualmente, as casas de alta-costura também costumam fazer coleções de prêt-à-porter com o intuito de divulgar a marca e aumentar o seu prestígio como símbolo de consumo de luxo (FISCHER, 2010).

A produção de peças feitas por meio do sistema de prêt-à-porter continua forte, porém, de acordo com Conti (2008), no final do século XX e início do século XXI, iniciou-se um sistema chamado de post-fashion (pós-moda). Neste sistema, se discutem os processos e a velocidade de produção, o consumo consciente e o papel da tecnologia inserida neste meio (JOY et al., 2012). Dentre os sistemas chamados por Conti (2008) de post-fashion, está o fast-fashion. De acordo com Cietta (2010), por volta do fim década de 1990, a maioria das grandes empresas do setor adotou o fast-fashion como modelo de produção de peças de vestuário, em uma tentativa de corresponder aos desejos dos consumidores cada vez mais exigentes e ansiosos por novidades.

O fast-fashion é um modelo de funcionamento do sistema da Moda que desenvolve artigos projetados baseando-se em tendências de consumo instantâneas. Trata-se, de um exemplo que revoluciona a tradicional maneira de apresentar as coleções sazonais, com um ciclo contínuo, e extremamente rápido de divulgar minicoleções, geralmente semanais. Para que este sistema funcione, é necessário que exista uma complexa estratégia de organização e gerência que reúne várias áreas de uma mesma empresa, garantindo velocidade de resposta do público consumidor por meio de uma venda rápida e assertiva (CIETTA, 2010). Porém, o sistema atual de fast-fashion não é de natureza autoral, geralmente não possui estética inovadora, além de ser produzida geralmente por processos insustentáveis (JOY et al., 2012). No próximo subcapítulo são descritas quais as relações entre o sistema da Moda e a tecnologia.

\subsection{O sistema da Moda e a tecnologia}

O sistema da Moda está extremamente ligado à evolução do capitalismo e ao avanço tecnológico (NOVIK; BLANCH, 2008). A utilização constante da internet e os progressos na logística e nos transportes permitiram a globalização e a multiculturalismo da Moda, deste modo, entendese que a tecnologia tem papel fundamental no crescimento da produção e comercialização de produtos e serviços ligados ao consumo de produtos de Moda (ARAÚJO; NEVES; NEVES, 2008; NECYK; FARBIARZ; FARBIARZ, 2016).

A partir desta lógica, nesta seção serão discutidas as formas como a tecnologia se faz presente nas fases de formação do sistema da Moda, nas etapas dos processos projetuais, que vão desde a definição das relações entre o público e a marca, passando por todo o processo de planejamento, desenvolvimento e produção até os meios de divulgação, comercialização e distribuição dos artigos de Moda.

Conforme Novik e Blanch (2008), a Moda, o Design, o Marketing e a publicidade são 
fenômenos sociais pertencentes à sociedade industrial capitalista. Seus diversos aspectos influenciam os âmbitos social, cultural, econômico e ambiental das mais variadas formas. Sendo assim, o surgimento da internet fez com que a disseminação de informações sobre produtos e serviços de consumo chegasse até os consumidores de forma avassaladora e definitiva.

$\mathrm{Na}$ etapa em que se constroem as relações entre o público consumidor com a marca, o Marketing tem ligação tanto com a segmentação de mercado como com a criação da identidade da marca. É neste ponto que se destacam as tecnologias utilizadas nas pesquisas de públicos alvo e os sistemas CAD-Computer Aided Design (desenho assistido por computador) específicos para a construção da identidade visual da marca, incluindo a tipografia e o logotipo, sistemas estes utilizados pelos designers gráficos em seus processos de pesquisa e criação (TREPTOW, 2013).

Treptow (2013) afirma que a pesquisa de Moda é realizada buscando conhecer os concorrentes do segmento de cada empresa, fazer a pesquisa das tendências e iniciar o planejamento da coleção. Porém, não basta conhecer somente o que o mercado já consome, o foco central da pesquisa de Moda é buscar compreender o comportamento do público consumidor, seus hábitos de vida e consumo e, especialmente os seus anseios. Nesta fase a tecnologia é indispensável, pois geralmente estas pesquisas são feitas via sites especializados em conteúdo online, os quais são atualizados constantemente, além de aplicativos e redes sociais onde há interação direta com o público-alvo da marca.

O planejamento da coleção é o momento em que é possível observar que a tecnologia se tornou mais indispensável, especialmente no que se refere à escolha dos tecidos e aviamentos utilizados na fabricação das peças de vestuário e acessórios. Araújo, Neves e Neves (2008) afirmam que um importante passo no desenvolvimento da tecnologia para a produção de produtos têxteis se deu com o crescimento científico significativo nas áreas de microeletrônica, nanotecnologia e biotecnologia.

De acordo com Seymour (2008), atualmente, com a implantação de tecnologia de ponta e a colaboração interdisciplinar entre cientistas, tecnólogos e designers as chamadas wearable (tecnologias vestíveis) também estão se tornando realidade. Desta maneira, o uso de materiais têxteis aprimorados em conjunto com as pesquisas em conjunto com engenharia, computação física e redes de comunicação sem fio e a medicina, está levando a Moda à essencial integração da função e do design estético.

A expansão destes campos interfere diretamente na produção de tecidos inteligentes e na criação do design de superfície, especialmente com a utilização de sistemas $C A D$, visando maior agilidade na produção, grande possibilidade de geração de alternativas e diminuição de custos na produção de padronagens e estampas (Araújo; Neves; Neves, 2008).

Ainda, relacionada à fase de planejamento de coleção, a tecnologia é indispensável à criação de desenhos estilizados e técnicos. De acordo com Treptow (2013), a partir dos anos 80 os sistemas CAD começaram a ser utilizados por designers de Moda para agilizar e dar mais precisão ao processo de criação de desenhos, seja utilizando as técnicas de desenhos vetorizados ou por bitmap. Atualmente os sistemas não são utilizados somente por meio de computadores, mas também via tablets e smartphones, tornando o processo dinâmico, ágil e produtivo.

$\mathrm{Na}$ etapa de desenvolvimento da coleção o emprego da tecnologia é imprescindível. Os sistemas CAD estão presentes no momento do desenvolvimento das fichas técnicas de produto, 
na criação da modelagem e nos processos de encaixe, risco e corte dos tecidos. Na etapa da modelagem é possível realizar o processo diretamente nos softwares ou digitalizar os moldes físicos já produzidos anteriormente, além de já ser possível criar ou alterar a modelagem em sistemas CAD 3D, otimizando esse processo tão complexo e demorado. Na fase de corte, já é possível contar com equipamentos que realizam os processos automaticamente por meio de máquinas com corte à laser ou com uma pequena faca, proporcionando precisão e qualidade às peças produzidas.

Ainda na fase de desenvolvimento de coleção, está a produção das peças, sendo as etapas de preparação e de costura. A tecnologia fez com que este processo evoluísse bastante desde a criação das primeiras máquinas de costura, com a ampla popularização e utilização de máquinas eletrônicas. Porém, na maioria dos casos ainda é necessário que o maquinário seja operado por um profissional, assim como acontecia no século XIX quando Isaac Singer criou a primeira máquina de costura (TREPTOW, 2013).

Certamente nas fases de divulgação, comercialização e distribuição dos produtos de Moda é que a presença fundamental da tecnologia é percebida mais nitidamente. Atualmente, das grifes de luxo de alta-costura até as marcas de fast-fashion, a divulgação dos desfiles, catálogos e vitrines das marcas é feita de forma digital por meio de websites, aplicativos para smartphones e redes sociais. Além da divulgação, a venda dos produtos ocorre principalmente via internet, modificando assim a relação entre a cultura de consumo e a Moda. A seguir, é apresentada uma discussão sobre a relação entre a Moda, o consumo e a tecnologia.

\section{ANÁLISE DA RELAÇÃO ENTRE MODA, CONSUMO E TECNOLOGIA}

A metodologia utilizada para realizar a pesquisa é a qualitativa, pois, busca entender fenômenos e situações proporcionando profundidade aos dados analisados por meio de experiências únicas (SAMPIERI; COLLADO; LUCIO, 2013). Quanto ao método, a pesquisa é descritiva, pois tem o intuito de descrever e investigar situações e fenômenos. O foco da pesquisa se dá por meio de reflexões teóricas e análises de como ocorre atualmente a relação entre o sistema da Moda, a cultura de consumo e a tecnologia.

Partindo das reflexões feitas até o momento, por meio das seções anteriores, é possível compreender que a cultura e a tecnologia são campos distintos, mas que se relacionam constantemente, especialmente no que se refere à indústria cultural pautada no consumo de bens e serviços (BAUDRILLARD, 2008; ADORNO; HORKHEIMER, 1985). Por sua vez, a Moda é considerada um dispositivo da sociedade de consumo de massa, na qual quase todo o seu sistema atual está vinculado à produção industrial em grande escala, suprindo necessidade de grandes grupos de consumidores. Diante das discussões realizadas, é possível observar que existe um paradoxo no que tange o sistema atual de produção e consumo de Moda, especialmente em relação aos avanços tecnológicos.

De um lado, entende-se que a tecnologia tornou os sujeitos totalmente submissos à cultura de consumo, perdendo a sua identidade e o direito de escolha, ficando à mercê das grandes massas. É possível observar este processo de consumo de coletividade, no sistema da Moda, mediante a implantação e continuação do movimento do prêt-à-porter e mais recentemente do 
fast-fashion, em que se busca a produção e a comercialização de artigos de Moda com a maior velocidade possível deixando de lado, na maioria das vezes, critérios como qualidade dos produtos, responsabilidade social, ambiental e econômica, incentivando o consumo desenfreado de peças temporais e praticamente descartáveis (JOY et al., 2012).

De outro modo, a tecnologia contribui com a ampliação da produção de produtos com mais valor agregado e formas de divulgação mais eficientes. Este é o caso da produção de tecidos inteligentes, das tecnologias vestíveis, da expansão das criações no design de superfície, bordados e modelagens (CONTI, 2008; SEYMOUR, 2008). É perceptível que estes processos agregam valor às peças e estão sendo retomados com a volta de peças feitas sob medida feitas nos ateliês e os movimentos que defendem um sistema produtivo menos acelerado e mais consciente do ponto de vista social, ambiental e econômico, como o slow-fashion (moda lenta).

Conforme Nishimura e Gontijo (2016), o slow-fashion é um movimento de Moda que visa a conscientização da redução do ritmo produtivo das peças de vestuário, com o objetivo de obter peças com conceito sustentável. O movimento do slow-fashion confronta o modelo atual de produção em massa do fast-fashion. Esse novo sistema, focado na redução da velocidade do processo produtivo e do consumo e pautado na preocupação com o meio ambiente e com o retorno social, busca valorizar o trabalho artesanal e local sem abrir mão do auxílio da tecnologia para o seu desenvolvimento.

Na última década, a sustentabilidade e a conduta ética começaram a se tornar importantes para os consumidores de Moda. Observando a chegada deste novo comportamento de consumo de bens e serviços, as empresas perceberam que a Moda acessível e com propósito virou tendência (JOY et al., 2012). Diante deste cenário atual, Novik e Blanch (2008), afirmam que é possível observar que há somente uma alternativa para o futuro do sistema da Moda: a transformação da consciência social, voltada à busca do equilíbrio humano, ecológico e de consumo consciente.

Neste cenário, a tecnologia tem papel fundamental para que haja a disseminação das informações, tanto no que se refere às marcas como no fornecimento de matéria prima e serviços, tendo como essência a produção de Moda consciente e a retomada da força da identidade dos sujeitos. Assim, surge uma nova e poderosa matriz que vincula inovação, Design, empreendedorismo e sustentabilidade (NOVIK; BLANCH, 2008).

\section{CONSIDERAÇÕES FINAIS}

A Moda se caracteriza por um sistema de consumo extremamente complexo, ainda muito baseado no processo de distinção e imitação de sujeitos e classes defendido por Simmel (2005) e Lipovetsky (2009). Murphie e Potts (2003) afirmam que há um grande impacto cultural gerado pela tecnologia diante dos indivíduos e dos grupos sociais, enfatizando assim, que a cultura e a tecnologia têm uma relação extremamente interligada, porém complexa.

Para que os sistemas sociais funcionem adequadamente, é necessário que haja uma organização industrial e tecnológica focada na produção e distribuição de produtos e na prestação de serviços eficientes (MURPHIE; POTTS, 2003). Deste modo, a tecnologia é utilizada em praticamente todos os setores da indústria e comércio de bens e serviços, em especial, no campo 
do Design e da Moda.

Para compreender como o complexo sistema da Moda é organizado, baseando-se na lógica social, a história de Moda é dividida em três importantes fases: alta-costura, prêt-à-porter e o post-fashion. Esses sistemas estabelecem uma relação contínua entre vida social, consumo e a indústria produtora de artefatos. Meios estes, em que a tecnologia é parte indispensável para a pesquisa, produção, divulgação e manutenção do sistema da Moda (CONTI, 2008; TREPTOW, 2013; FISCHER, 2010; CIETTA, 2010; JOY et al., 2012).

Ao analisar a relação entre o sistema da Moda, a cultura de consumo e a tecnologia é possível afirmar que há a possibilidade de interação entre estes complexos e paradoxos campos. Assim, entende-se que a tecnologia pode ser utilizada para impulsionar a indústria da Moda rumo aos seus avanços, questionando a dominação da cultura de massa, não perdendo dessa forma, a essência da identidade e individualidade dos sujeitos (NOVIK; BLANCH, 2008).

Entende-se também, que a Moda possui funções físicas, culturais e psicológicas e, por meio da tecnologia as atribuições da roupa podem ser aprimoradas e novos usos são definidos. Deste modo, as tecnologias incorporadas ao vestuário influenciam a usabilidade, o conforto e a estética, sendo possível observar o alto grau de integração entre os indivíduos e a tecnologia empregada na Moda, onde o corpo está se tornando rapidamente a extensão para os avanços técnicos (SEYMOUR, 2008).

Além disso, compreende-se que a tecnologia pode auxiliar, por meio de pesquisas e divulgação, no processo atual de valorização da Moda autoral, autêntica e ética. Assim, atuando concomitantemente nos processos de inovação, valorização dos produtores de artefatos e do respeito e preservação ao meio ambiente, representando assim, uma revolução no sistema da Moda (JOY et al., 2012). 


\section{Referências}

ADORNO, Theodor W.; HORKHEIMER, MAX. Dialética do esclarecimento: fragmentos filosóficos. Rio de janeiro: Jorge Zahar, 1985. BIDERMAN, C.; COZAC, L. F. L.; REGO, J. M. Conversas com economistas brasileiros. 2.ed. São Paulo: Ed. 34, 1997.

ARAÚJO, Mário; NEVES, Manuela; NEVES, Jorge. O impacto da globalização e do multiculturalismo no design de moda. In. PIRES, Dorotéia B. Design de moda: olhares diversos / Dorotéia Baduy Pires (org). Barueri: Estação das Letras e Cores, 2008.

BAUDRILLARD, Jean. A sociedade de consumo. Portugal: Edições 70, [1970] 2008.

CIETTA, Enrico. A revolução do fast fashion. Estratégias e modelos organizativos para competir nas indústrias híbridas. São Paulo: Estação das Letras e Cores, 2010.

CONTI, Giovanni M. Moda e cultura de projeto industrial: hibridação entre saberes complexos. In. PIRES, Dorotéia B. Design de moda: olhares diversos / Dorotéia Baduy Pires (org). Barueri: Estação das Letras e Cores, 2008.

FISCHER, Anette. Fundamentos de design de moda: construção de vestuário. Porto Alegre: Bookman, 2010.

JOY, Annamma; SHERRY, John F.; VENKATESH, Alladi; WANG, Jeff; CHAN, Ricky. Fast Fashion, Sustainability, and the Ethical Appeal of Luxury Brands. Fashion Theory, Volume 16, Issue 3, pp. 273-296, 2012. Disponível em: < https://goo.gl/3oNfJW >. Acesso em: 06 fev. 2018.

LIPOVETSKY, Gilles. O império do efêmero: a moda e seu destino nas sociedades modernas. São Paulo: Companhia de Bolso, 2009.

MURPHIE, Andrew; POTTS, John. Culture and technology. Londres: Palgrave, 2003.

NECYK, Barbara J.; FARBIARZ, Jackeline L.; FARBIARZ, Alexandre. Design e mediação tecnológica: formas de produção de subjetividade. Anais do 12 ㅇ Congresso Brasileiro de Pesquisa e Desenvolvimento em Design. Belo Horizonte, 2016.

NISHIMURA, Maicon D. L.; GONTIJO, Leila A. Slow fashion e o produto de moda com enfoque no usuário. Anais do 12 을 Congresso Brasileiro de Pesquisa e Desenvolvimento em Design. Belo Horizonte, 2016.

NOVIK, Laura; BLANCH, Alex. Design, moda e negócios politicamente corretos. In. PIRES, Dorotéia B. Design de moda: olhares diversos / Dorotéia Baduy Pires (org). Barueri: Estação das Letras e Cores, 2008.

SAMPIERI, Roberto H.; COLLADO, Carlos F.; LUCIO, María del Pilar B. Metodologia de pesquisa. 5. ed. Porto Alegre: Penso, 2013.

SEYMOUR, Sabine. Fashionable Technology: The Intersection of Design, Fashion, Science and Technology. New York: SpringerWien, 2008.

SIMMEL, Georg. Da psicologia da moda: um estudo sociológico. In: SOUZA, Jessé; OELZE, Berthold. Simmel e a modernidade. Brasília: UnB, [1902] 2005.

TREPTOW, Doris. Inventando moda: planejamento de coleção. 5. ed. São Paulo: Edição da Autora, 2013. 